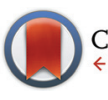

CrossMark \& click for updates

Cite this: Org. Biomol. Chem., 2015, 13,6493

Received 7th April 2015,

Accepted 27th April 2015

DOI: $10.1039 / \mathrm{c} 5 \mathrm{ob} 00688 \mathrm{k}$

www.rsc.org/obc

\title{
Discovery of novel isatin-based sulfonamides with potent and selective inhibition of the tumor- associated carbonic anhydrase isoforms IX and XII
}

\author{
Özlen Güzel-Akdemir, ${ }^{a}$ Atilla Akdemir, ${ }^{\mathrm{b}}$ Nilgün Karalı ${ }^{\mathrm{a}}$ and Claudiu T. Supuran*c
}

A series of 2/3/4-[(2-oxo-1,2-dihydro-3H-indol-3-ylidene)amino]benzenesulfonamides, obtained from substituted isatins and 2-, 3- or 4-aminobenzenesulfonamide, showed low nanomolar inhibitory activity against the tumor associated carbonic anhydrase (CA, EC 4.2.1.1) isoforms IX and XII - recently validated antitumor drug targets, being much less effective as inhibitors of the off-target cytosolic isoforms CA I and II.

\section{Introduction}

Carbonic anhydrases (CAs, EC 4.2.1.1) are widespread metalloenzymes with catalytic versatility, having as substrates $\mathrm{CO}_{2}$, $\mathrm{COS}, \mathrm{CS}_{2}$, cyanamide, carboxylic, phosphoric and thiocarboxylic esters. ${ }^{1-4}$ Although their main physiological functions are related to the inter-conversion between $\mathrm{CO}_{2}$, bicarbonate and protons, they play crucial functions in $\mathrm{pH}$ regulation, electrolyte secretion, biosynthetic reactions, and carcinogenesis, among others. ${ }^{5-8}$ Of the 15 isoforms described in humans (hCA I-XIV), many are drug targets for pharmacological agents such as diuretics, ${ }^{9}$ antiglaucoma drugs,${ }^{10}$ antiepileptics, ${ }^{11}$ antiobesity $^{12}$ and ultimately, antitumor drugs/cancer diagnostic agents. ${ }^{13}$ The transmembrane isoforms CA IX and XII are overexpressed in hypoxic tumors as a consequence of the HIF-1 $\alpha$ (hypoxia inducible factor-1 $\alpha$ ) activation pathway, and their inhibition by small molecules/antibodies was recently shown to lead to significant antitumor action. ${ }^{13,14}$ Furthermore, as these enzymes are present in normal tissues in a very small concentration compared to the tumors, they can also be used for imaging hypoxic tumors. ${ }^{15}$ Recently, a sulfonamide CA inhibitor (CAI) targeting CA IX and XII entered phase I clinical trials for the treatment of advanced metastatic solid tumors. ${ }^{16}$

\footnotetext{
${ }^{a}$ Istanbul University, Faculty of Pharmacy, Department of Pharmaceutical Chemistry, 34116 Beyazlt, Istanbul, Turkey

${ }^{b}$ Bezmialem Vakif University, Faculty of Pharmacy, Department of Pharmacology, Vatan Caddesi, 34093, Fatih, Istanbul, Turkey.E-mail: aakdemir@bezmialem.edu.tr ${ }^{c}$ Universita degli Studi di Firenze, NEUROFARBA Dept., Sezione di Scienze Farmaceutiche, Via Ugo Schiff 6, 50019 Sesto Fiorentino (Florence), Italy. E-mail: claudiu.supuran@unifi.it
}

\section{Results and discussion}

\section{Chemistry}

Sulfonamides $\left(\mathrm{RSO}_{2} \mathrm{NH}_{2}\right)$ constitute the most important and investigated class of CAIs. ${ }^{17}$

A large number of structurally diverse sulfonamides were investigated for their CA inhibitory properties. ${ }^{17,18}$ However the main problem with sulfonamides is their promiscuous behavior as strong inhibitors of many of the $15 \mathrm{CA}$ isoforms of human (h) origin. ${ }^{17,18}$ As isoforms hCA I and II are widespread and play important physiological functions, ${ }^{1-3}$ it is of great interest to design inhibitors targeting the tumor-associated isoforms hCA IX and XII, which, at the same time, show weak affinity for the off-target isoforms hCA I and II. Some Schiff bases incorporating sulfonamide moieties were among the first types of CAIs showing selective inhibition of some CA isoforms of interest for medicinal chemistry applications, ${ }^{19,20}$ and this is the reason why we explore here these types of compounds which incorporate substituted isatin moieties (Scheme 1). Reaction of isatins with aromatic sulfonamides was in fact investigated earlier by our and other groups, ${ }^{21-25}$ and a limited number of such compounds have been reported. Here we extend the previous studies, reporting a series of 23 such derivatives which incorporate orthanilamide, metanilamide or sulfanilamide moieties, as well as isatin or $\mathrm{N}$-methylisatins substituted with methyl, halogens, nitro or trifluoromethyloxy moieties at the heterocyclic ring. We have chosen these substitution patterns at the isatin fragment of the molecule in order to investigate the structure-activity relationship (SAR) for the inhibition of four CA isoforms (hCA I, II, IX and XII) with this class of derivatives. ${ }^{26}$

\section{Enzyme inhibition data}

hCA I was inhibited moderately by the reported compounds irrespective of the substitution pattern, with $K_{\mathrm{I}} \mathrm{S}$ ranging 

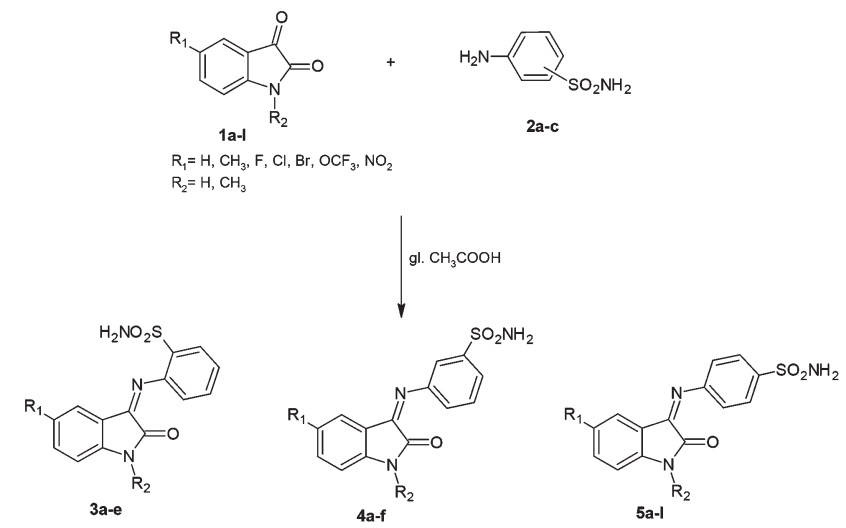

Scheme 1 Preparation of 2/3/4-[(2-oxo-1,2-dihydro-3H-indol-3ylidene)amino]benzenesulfonamides.

between 146 and $816 \mathrm{nM}$. The same was true for the cytosolic dominant isoform hCA II; for which the inhibition constants were in the range of 101-728 nM (Table 1). All $K_{\mathrm{I}}$ values were in the lower nanomolar region, in a narrow range, for both tumor-associated hCA isozymes (hCA IX: 1.0-15.6 nM; hCA XII: 2.8-53.8 nM; Table 1). The $K_{\mathrm{I}}$ values for the widespread hCA I/II were significantly larger (hCA I: 146-816 nM; hCA II: 101-728 nM) and thus, the new compounds showed a discrete selectivity for the tumor-associated isozymes (Table 1). The difference in $K_{\mathrm{I}}$ values is relatively small for the tumor-associated isozymes (hCA IX: 15-fold; hCA XII: 19-fold) and a conclusive SAR analysis is difficult to perform. Compound $\mathbf{4 b}$ drew our attention since it shows very low $K_{\mathrm{I}}$ values for the tumor-associated isozymes (hCA IX: $1.1 \mathrm{nM}$; hCA XII: $3.3 \mathrm{nM}$ ) and it shows the highest selectivity for the tumor-associated isozymes compared to the widely distributed hCA I and II (Table 1).

\section{Molecular modelling studies}

Compound $\mathbf{4 b}$ has one of the lowest measured $K_{\mathrm{I}}$ values for hCA IX and shows the highest selectivity towards hCA IX compared to the other isozymes (Table 1). Molecular modelling studies were applied to suggest a rationale for this selectivity. Available crystal structures of hCA isozymes with sulfonamidecontaining ligands such as acetazolamide bound to their active site indicate that the sulfonamide moiety is oriented in a very similar way to the $\mathrm{Zn}^{2+}$-ion of the hCA active sites. The nitrogen atom of the $\mathrm{SO}_{2} \mathrm{NH}^{-}$group is coordinated to the $\mathrm{Zn}^{2+}$-ion and forms a hydrogen bond with the side-chain of Thr199, whereas one of the sulfonamide oxygen atoms forms a hydrogen bond with the backbone $\mathrm{NH}$ of the same residue. A similar orientation and binding-interactions were enforced upon the ligands in our docking studies.

Docking studies on hCA IX. The docked pose of compound $\mathbf{4 b}$ in the active site of hCA IX reveals that the vicinal nitrogen and carbonyl group of the indole ring form hydrogen bonds with the side-chains of Gln67 and Gln92, respectively (Fig. 1). The other analogs with sulfonamide groups on the meta position of the phenyl ring (compound series 4) adopted similar docked poses and the range of $K_{\mathrm{I}}$ values was 1.1-9.8 nM. The various substituents on the isatin ring did not form any

Table 1 hCA I, II, IX and XII inhibition data of compounds 3, 4 and 5 by a stopped-flow $\mathrm{CO}_{2}$ hydrase assay ${ }^{26}$

\begin{tabular}{|c|c|c|c|c|c|c|c|c|c|c|}
\hline \multicolumn{3}{|c|}{ Compounds } & \multicolumn{4}{|c|}{$K_{\mathrm{I}}(\mathrm{nM})$} & \multicolumn{4}{|c|}{ Selectivity ratio } \\
\hline $3 \mathbf{b}$ & $\mathrm{CH}_{3}$ & $\mathrm{H}$ & 600 & 711 & 4.2 & 49.7 & 143 & 169 & 12 & 14 \\
\hline $3 c$ & $\mathrm{~F}$ & $\mathrm{H}$ & 652 & 428 & 1.2 & 41.3 & 543 & 357 & 16 & 10 \\
\hline $3 d$ & $\mathrm{Cl}$ & $\mathrm{H}$ & 778 & 652 & 5.7 & 52.5 & 136 & 114 & 15 & 12 \\
\hline $3 e$ & $\mathrm{OCF}_{3}$ & $\mathrm{H}$ & 742 & 683 & 10.3 & 53.0 & 72 & 66 & 14 & 13 \\
\hline $4 c$ & $\mathrm{~F}$ & $\mathrm{H}$ & 426 & 264 & 1.3 & 30.8 & 328 & 203 & 14 & 9 \\
\hline 4d & $\mathrm{Cl}$ & $\mathrm{H}$ & 490 & 547 & 4.8 & 41.1 & 102 & 114 & 12 & 13 \\
\hline $4 e$ & $\mathrm{OCF}_{3}$ & $\mathrm{H}$ & 539 & 484 & 9.8 & 36.5 & 55 & 49 & 15 & 13 \\
\hline 4f & $\mathrm{NO}_{2}$ & $\mathrm{H}$ & 378 & 250 & 5.7 & 3.1 & 66 & 44 & 122 & 81 \\
\hline $5 a$ & $\mathrm{H}$ & $\mathrm{H}$ & 422 & 523 & 6.5 & 29.3 & 65 & 80 & 14 & 18 \\
\hline $5 b$ & $\mathrm{H}$ & $\mathrm{CH}_{3}$ & 586 & 549 & 5.9 & 6.7 & 99 & 93 & 87 & 82 \\
\hline $5 \mathrm{~h}$ & $\mathrm{Cl}$ & $\mathrm{CH}_{3}$ & 368 & 462 & 4.7 & 44.6 & 78 & 98 & 8 & 10 \\
\hline $5 i$ & $\mathrm{Br}$ & $\mathrm{CH}_{3}$ & 457 & 514 & 15.6 & 37.8 & 29 & 33 & 12 & 14 \\
\hline $5 \mathbf{j}$ & $\mathrm{OCF}_{3}$ & $\mathrm{H}$ & 295 & 236 & 3.9 & 3.8 & 76 & 61 & 78 & 62 \\
\hline $5 \mathbf{k}$ & $\mathrm{NO}_{2}$ & $\mathrm{H}$ & 229 & 164 & 4.9 & 2.8 & 47 & 33 & 82 & 59 \\
\hline 51 & $\mathrm{NO}_{2}$ & $\mathrm{CH}_{3}$ & 146 & 101 & 4.2 & 4.3 & 35 & 24 & 34 & 23 \\
\hline $\mathrm{AZ}$ & - & - & 250 & 12 & 25.0 & 5.7 & 10 & 0 & 44 & 2 \\
\hline
\end{tabular}




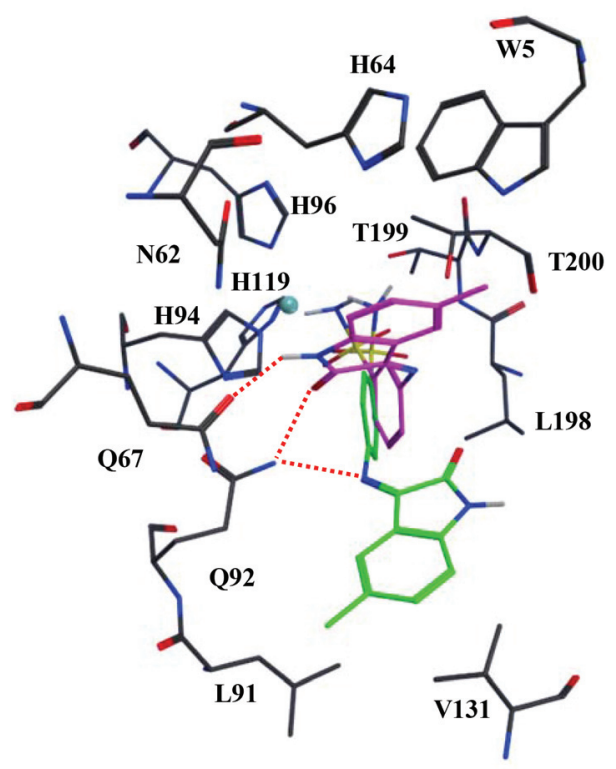

Fig. 1 The docked poses of compounds $4 b$ (magenta) and $5 c$ (green) in the active site of hCA IX. Interactions of the sulfonamide group with the protein are not shown for clarity. Hydrogen bonds are indicated with red dashed lines. The $\mathrm{Zn}^{2+}$-ion is indicated with a turquoise sphere.

additional interactions except for compound $\mathbf{4 f}$, which has a $\mathrm{NO}_{2}$ group capable of forming a hydrogen bond with Trp5.

Compound $\mathbf{5 c}$ is very similar to $\mathbf{4 b}$, except for the fact that the sulfonamide is substituted on the para position instead of the meta position. This reorients the isatin fragment to form hydrogen bonds with Gln92 via the imine group between the 2-indolinone and the phenyl ring (Fig. 1). In addition, hydrophobic interactions were observed between the isatin moiety and the sidechain of Val131. The analogs with a sulfonamide in the para position (compound series 5) showed a similar docked pose. Their range of $K_{\mathrm{I}}$ values is $1.0-15.6 \mathrm{nM}$ and the varying substituents do not form additional interactions with the active site, as they point towards the solvent.

Differences in active sites between hCA IX and hCA XII. Gln67 and Gln92 are involved in hydrogen bonding to compound $\mathbf{4 b}$ and are believed to be responsible for the low $K_{\mathrm{I}}$ value observed for this compound. Gln67 is not conserved amongst the other hCA isozymes (Table 2). hCA XII has a Lys67 instead of the Gln67 of hCA IX and the ligand cannot form the same interactions as observed in Fig. 1. Gln92 is conserved in both structures and the backbone is located at a similar position, but the sidechain conformation is slightly different. As such, no hydrogen bond is observed in the docking, but it should be possible after sidechain reorientation.

Differences in active sites between hCA IX and hCA I. The bulky His67 is present in hCA I instead of the Gln67 of hCA IX (Table 2). In addition, Val131 and Thr200 of hCA IX are replaced by the larger Leu131 and His200 in hCA I (Table 2). The presence of His 200 forces a reorientation of Trp5, which
Table 2 The differences and similarities in the active site lining of the investigated hCA isozymes

\begin{tabular}{llll}
\hline hCA IX & hCA XII & hCA I & hCA II \\
\hline Trp5 $^{a}$ & Trp5 & Trp5 & Trp5 \\
Gln67 $^{a}$ & Lys67 & His67 & Asn67 \\
Gln92 $^{a}$ & Gln92 & Gln92 & Gln92 \\
Val131 & Ala131 & Leu131 & Phe131 \\
Thr199 & Thr199 & His200 & Thr199
\end{tabular}

${ }^{a}$ This residue is conserved but has a different orientation in at least one crystal structure.

enters the active site more deeply and sterically interferes with the docked ligands. These changes in the binding site do not allow for the adoption of similar poses as observed in Fig. 1 .

Differences in active sites between hCA IX and hCA II. Asn67 and Phe131 are present in hCA II (Table 2). Asn67 is shorter than its Gln67 counterpart observed in hCA IX, while Phe131 points into the active site to a larger degree compared to Val131.

\section{Experimental}

\section{Synthetic procedures}

Melting points were estimated with a Buchi 540 melting point apparatus in open capillaries and are uncorrected. Elemental analyses were performed on a Thermo Finnigan Flash EA 1112 elemental analyzer. IR spectra were recorded on $\mathrm{KBr}$ discs, using a Perkin-Elmer Model 1600 FT-IR spectrometer. ${ }^{1} \mathrm{H}-\mathrm{NMR}, \mathrm{D}_{2} \mathrm{O}$-exch., HSQC and HMBC spectra were obtained

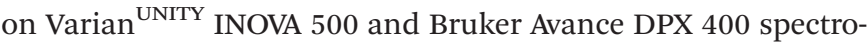
photometers using DMSO- $d_{6}$.

Synthesis of 2/3/4-[(2-oxo-1,2-dihydro-3H-indol-3-ylidene)amino]benzenesulfonamides (3a-e, 4a-f, 5a-1). Equimolar quantities of $1 H$-indole-2,3-diones (1) $(0.01 \mathrm{~mol})$ and 2-aminobenzenesulfonamide/3-aminobenzenesulfonamide/4-aminobenzenesulfonamide (2) were refluxed in glacial acetic acid $(10 \mathrm{ml})$ for $6 \mathrm{~h}$. The reaction mixture was allowed to stand for $24 \mathrm{~h}$ at room temperature. The product was filtered and recrystallized from ethanol. ${ }^{21}$

2-[(2-Oxo-1,2-dihydro-3H-indol-3-ylidene)amino]benzenesulfonamide (3a). Yellow powder, yield $66 \%$; m.p. $249-250{ }^{\circ} \mathrm{C}$; IR $(\mathrm{KBr})\left(\nu, \mathrm{cm}^{-1}\right): 3291,3180(\mathrm{NH}), 1735(\mathrm{C}=\mathrm{O}), 1337,1151$ $(\mathrm{S}=\mathrm{O}) ;{ }^{1} \mathrm{H}-\mathrm{NMR}$ (DMSO- $\left.d_{6}, 400 \mathrm{MHz}\right) \delta(\mathrm{ppm}): 6.68-8.25(10 \mathrm{H}$, $\left.\mathrm{m}, \mathrm{Ar}-\mathrm{H}, \mathrm{SO}_{2} \mathrm{NH}_{2}\right), 10.58,10.96(1 \mathrm{H}, 2 \mathrm{~s}$, indole $\mathrm{NH})$. Anal. Calcd for $\mathrm{C}_{14} \mathrm{H}_{11} \mathrm{~N}_{3} \mathrm{O}_{3} \mathrm{~S}$ (301.32): C, 55.80, H, 3.68; N, 13.95; S, 10.64. Found: C, 55.51; H, 3.94; N, 13.84; S, 10.50 .

2-[(5-Methyl-2-oxo-1,2-dihydro-3H-indol-3-ylidene)amino]benzenesulfonamide (3b). Orange powder, yield 24\%; m.p. $235-236{ }^{\circ} \mathrm{C}$; IR $(\mathrm{KBr})\left(\nu, \mathrm{cm}^{-1}\right): 3274,3192(\mathrm{NH}), 1732$ $(\mathrm{C}=\mathrm{O}), 1327,1160(\mathrm{~S}=\mathrm{O}) ;{ }^{1} \mathrm{H}-\mathrm{NMR} \quad\left(\mathrm{DMSO}_{6} d_{6}, 500 \mathrm{MHz}\right)$ $\delta(\mathrm{ppm}): 1.94,2.29\left(3 \mathrm{H}, 2 \mathrm{~s}, 5-\mathrm{CH}_{3}\right), 6.09-7.65(6 \mathrm{H}, \mathrm{m}, \mathrm{Ar}-\mathrm{H})$, 6.94, $7.03\left(2 \mathrm{H}, 2 \mathrm{~s}, \mathrm{SO}_{2} \mathrm{NH}_{2}\right), 7.80,7.95(1 \mathrm{H}, 2 \mathrm{~d}, J=7.81 \mathrm{~Hz}$, phenyl $\left.\mathrm{C}_{6}-\mathrm{H}\right), 10.68,10.86(1 \mathrm{H}, 2 \mathrm{~s}$, indole $\mathrm{NH})$. Anal. Calcd for 
$\mathrm{C}_{15} \mathrm{H}_{13} \mathrm{~N}_{3} \mathrm{O}_{3} \mathrm{~S}$ (315.34): C, 57.13, H, 4.16; N, 13.33; S, 10.17. Found: C, 57.55; H, 4.44; N, 13.14; S, 9.90.

2-[(5-Fluoro-2-oxo-1,2-dihydro-3H-indol-3-ylidene)amino]benzenesulfonamide (3c). Orange powder, yield 85\%; m.p. 269-271 ${ }^{\circ} \mathrm{C}$; IR (KBr) $\left(\nu, \mathrm{cm}^{-1}\right): 3290,3187(\mathrm{NH}), 1731(\mathrm{C}=\mathrm{O})$, 1338, $1168(\mathrm{~S}=\mathrm{O}) ;{ }^{1} \mathrm{H}-\mathrm{NMR}$ (DMSO- $\left.d_{6}, 500 \mathrm{MHz}\right) \delta$ (ppm): 6.81 $\left(1 \mathrm{H}, \mathrm{d}, J=7.32 \mathrm{~Hz}\right.$, phenyl $\left.\mathrm{C}_{3}-\mathrm{H}\right), 6.83(1 \mathrm{H}, \mathrm{t}, J=7.81 \mathrm{~Hz}$, phenyl $\left.\mathrm{C}_{5}-\mathrm{H}\right), 6.86\left(1 \mathrm{H}, \mathrm{dd}, J=8.29,4.39 \mathrm{~Hz}\right.$, indole $\left.\mathrm{C}_{7}-\mathrm{H}\right)$, $7.15\left(1 \mathrm{H}, \mathrm{dt}, J=9.26,2.93 \mathrm{~Hz}\right.$, indole $\left.\mathrm{C}_{6}-\mathrm{H}\right), 7.32-7.36(2 \mathrm{H}, \mathrm{m}$, indole $\mathrm{C}_{4}-\mathrm{H}$, phenyl $\left.\mathrm{C}_{4}-\mathrm{H}\right), 7.51(1 \mathrm{H}, \mathrm{dd}, J=7.81,0.98 \mathrm{~Hz}$, phenyl $\left.\mathrm{C}_{6}-\mathrm{H}\right), 7.64,8.36\left(2 \mathrm{H}, 2 \mathrm{~s}, \mathrm{SO}_{2} \mathrm{NH}_{2}, \mathrm{D}_{2} \mathrm{O}\right.$ exch.), 10.64 $\left(1 \mathrm{H}, \mathrm{s}\right.$, indole $\mathrm{NH}, \mathrm{D}_{2} \mathrm{O}$ exch.). HSQC (DMSO- $\left.d_{6}\right) \delta(\mathrm{ppm})$ : $111.73\left(\mathrm{~d}, J=7.66 \mathrm{~Hz}\right.$, indole $\left.\mathrm{C}_{7}\right), 114.36(\mathrm{~d}, J=25.87 \mathrm{~Hz}$, indole $\mathrm{C}_{4}$ ), 117.07 (phenyl $\mathrm{C}_{5}$ ), $117.53(\mathrm{~d}, J=23.48 \mathrm{~Hz}$, indole $\mathrm{C}_{6}$ ), 118.29 (phenyl $\mathrm{C}_{3}$ ), 123.42 (phenyl $\mathrm{C}_{6}$ ), 123.95 (phenyl $\mathrm{C}_{1}$ ), $130.98\left(\mathrm{~d}, J=8.15 \mathrm{~Hz}\right.$, indole $\mathrm{C}_{3 \mathrm{a}}$ ), 133.81 (phenyl $\mathrm{C}_{4}$ ), 138.10 (indole $\mathrm{C}_{7 \mathrm{a}}$ ), 138.11 (indole $\mathrm{C}_{3}$ ), 143.81 (phenyl $\mathrm{C}_{2}$ ), $158.50\left(\mathrm{~d}, J=237.71\right.$, indole $\mathrm{C}_{5}$ ), 174.00 (indole $\mathrm{C}_{2}$ ). Anal. Calcd for $\mathrm{C}_{14} \mathrm{H}_{10} \mathrm{FN}_{3} \mathrm{O}_{3} \mathrm{~S}$ (319.31): C, 52.66, H, 3.16; N, 13.16; S, 10.04. Found: C, 52.52; H, 3.13; N, 13.12; S, 10.11 .

2-[(5-Chloro-2-oxo-1,2-dihydro-3H-indol-3-ylidene)amino]benzenesulfonamide (3d). Orange powder, yield 40\%; m.p. 248-250 ${ }^{\circ} \mathrm{C}$; IR $(\mathrm{KBr})\left(\nu, \mathrm{cm}^{-1}\right): 3266,3197(\mathrm{NH}), 1731(\mathrm{C}=\mathrm{O})$, 1328, $1156(\mathrm{~S}=\mathrm{O}) ;{ }^{1} \mathrm{H}-\mathrm{NMR}$ (DMSO- $\left.d_{6}, 500 \mathrm{MHz}\right) \delta$ (ppm): 6.82 $\left(1 \mathrm{H}, \mathrm{t}, J=7.81 \mathrm{~Hz}\right.$, phenyl $\left.\mathrm{C}_{5}-\mathrm{H}\right), 6.89(1 \mathrm{H}, \mathrm{d}, J=8.29 \mathrm{~Hz}$, indole $\left.\mathrm{C}_{7}-\mathrm{H}\right), 7.35\left(1 \mathrm{H}\right.$, dd, $J=7.81,1.46 \mathrm{~Hz}$, phenyl $\left.\mathrm{C}_{3}-\mathrm{H}\right)$, $7.36\left(1 \mathrm{H}, \mathrm{dd}, J=8.29,2.44 \mathrm{~Hz}\right.$, indole $\left.\mathrm{C}_{6}-\mathrm{H}\right), 6.86,8.41(2 \mathrm{H}, 2 \mathrm{~s}$, $\mathrm{SO}_{2} \mathrm{NH}_{2}, \mathrm{D}_{2} \mathrm{O}$ exch.), $7.52\left(1 \mathrm{H}, \mathrm{dd}, J=7.81,2.44 \mathrm{~Hz}\right.$, phenyl $\mathrm{C}_{4}-$ $\mathrm{H}), 7.54\left(1 \mathrm{H}, \mathrm{d}, J=2.44 \mathrm{~Hz}\right.$, phenyl $\left.\mathrm{C}_{6}-\mathrm{H}\right), 7.64(1 \mathrm{H}$, s, indole $\left.\mathrm{C}_{4}-\mathrm{H}\right), 10.75\left(1 \mathrm{H}, \mathrm{s}\right.$, indole $\mathrm{NH}, \mathrm{D}_{2} \mathrm{O}$ exch.). Anal. Calcd for $\mathrm{C}_{14} \mathrm{H}_{10} \mathrm{ClN}_{3} \mathrm{O}_{3} \mathrm{~S}$ (335.76): C, 50.08, H, 3.00; N, 12.51; S, 9.55. Found: C, 49.77; H, 3.12; N, 12.35; S, 9.48.

2-[(2-Oxo-5-(trifluoromethoxy)-1,2-dihydro-3 $\mathrm{H}$-indol-3-ylidene)amino]benzenesulfonamide (3e). Orange powder, yield 30\%; m.p. 261-263 ${ }^{\circ} \mathrm{C}$; IR (KBr) $\left(\nu, \mathrm{cm}^{-1}\right)$ : 3289, $3187(\mathrm{NH}), 1733$ $(\mathrm{C}=\mathrm{O}), 1339,1163(\mathrm{~S}=\mathrm{O}) ;{ }^{1} \mathrm{H}-\mathrm{NMR} \quad\left(\mathrm{DMSO}-d_{6}, 500 \mathrm{MHz}\right)$ $\delta(\mathrm{ppm}): 6.82\left(1 \mathrm{H}, \mathrm{d}, J=8.29 \mathrm{~Hz}\right.$, indole $\left.\mathrm{C}_{7}-\mathrm{H}\right), 6.86(1 \mathrm{H}, \mathrm{d}, J=$ $7.81 \mathrm{~Hz}$, phenyl $\left.\mathrm{C}_{3}-\mathrm{H}\right), 6.95\left(1 \mathrm{H}, \mathrm{d}, J=8.29 \mathrm{~Hz}\right.$, indole $\left.\mathrm{C}_{6}-\mathrm{H}\right)$, $7.32\left(1 \mathrm{H}, \mathrm{dd}, J=8.29,1.95 \mathrm{~Hz}\right.$, indole $\left.\mathrm{C}_{4}-\mathrm{H}\right), 7.36(1 \mathrm{H}, \mathrm{dd}, J=$ 7.81, $1.46 \mathrm{~Hz}$, phenyl $\left.\mathrm{C}_{5}-\mathrm{H}\right), 7.52(2 \mathrm{H}, \mathrm{dd}, J=7.81,1.46 \mathrm{~Hz}$, phenyl $\left.\mathrm{C}_{4,6}-\mathrm{H}\right), 7.66,8.46\left(2 \mathrm{H}, 2 \mathrm{~s}, \mathrm{SO}_{2} \mathrm{NH}_{2}\right), 10.81(1 \mathrm{H}, \mathrm{s}$, indole $\mathrm{NH}$ ). HMBC (DMSO- $\left.d_{6}\right) \delta(\mathrm{ppm}): 111.86$ (indole $\mathrm{C}_{7}$ ), 117.10 (phenyl $\mathrm{C}_{5}$ ), 118.41 (indole $\left.\mathrm{C}_{4}\right), 119.49\left(\mathrm{OCF}_{3}\right), 120.57$ (indole $\mathrm{C}_{6}$ ), 123.37 (phenyl $\mathrm{C}_{3}$ ), 124.07 (phenyl $\mathrm{C}_{6}$ ), 124.46 (phenyl $\mathrm{C}_{1}$ ), 130.99 (indole $\mathrm{C}_{3 \mathrm{a}}$ ), 132.69 (indole $\mathrm{C}_{5}$ ), 133.86 (phenyl $\mathrm{C}_{4}$ ), 141.09 (indole $\mathrm{C}_{3}$ ), 143.73 (phenyl $\mathrm{C}_{2}$ ), 143.85 (indole $\mathrm{C}_{7 \mathrm{a}}$ ), 174.05 (indole $\mathrm{C}_{2}$ ). Anal. Calcd for $\mathrm{C}_{15} \mathrm{H}_{10} \mathrm{~F}_{3} \mathrm{~N}_{3} \mathrm{O}_{4} \mathrm{~S}$ (385.32): C, 46.76, H, 2.62; N, 10.91; S, 8.32. Found: C, 46.44; $\mathrm{H}, 2.84 ; \mathrm{N}, 10.56 ; \mathrm{S}, 8.32$.

3-[(2-Oxo-1,2-dihydro-3H-indol-3-ylidene)amino]benzenesulfonamide (4a). Yellow powder, yield 24\%; m.p. $263-264{ }^{\circ} \mathrm{C}$; IR $(\mathrm{KBr})\left(\nu, \mathrm{cm}^{-1}\right): 3361,3259(\mathrm{NH}), 1725,1746(\mathrm{C}=\mathrm{O}), 1330$, $1147(\mathrm{~S}=\mathrm{O}) ;{ }^{1} \mathrm{H}-\mathrm{NMR}$ (DMSO- $\left.d_{6}, 500 \mathrm{MHz}\right) \delta$ (ppm): 6.32-7.69 $(8 \mathrm{H}, \mathrm{m}, \mathrm{Ar}-\mathrm{H}), 7.33,7.41\left(2 \mathrm{H}, 2 \mathrm{~s}, \mathrm{SO}_{2} \mathrm{NH}_{2}\right), 10.88,10.98(1 \mathrm{H}$, 2s, indole $\mathrm{NH}$ ). Anal. Calcd for $\mathrm{C}_{14} \mathrm{H}_{11} \mathrm{~N}_{3} \mathrm{O}_{3} \mathrm{~S}$ (301.32): C, 55.80,
H, 3.68; N, 13.95; S, 10.64. Found: C, 55.89; H, 4.06; N, 13.64; S, 10.65 .

3-[(5-Methyl-2-oxo-1,2-dihydro-3H-indol-3-ylidene)amino]benzenesulfonamide (4b). Orange powder, yield 32\%; m.p. 243-245 ${ }^{\circ} \mathrm{C}$; IR (KBr) $\left(\nu, \mathrm{cm}^{-1}\right)$ : 3269, $3175(\mathrm{NH}), 1731(\mathrm{C}=\mathrm{O})$, 1326, $1154(\mathrm{~S}=\mathrm{O})$; ${ }^{1} \mathrm{H}-\mathrm{NMR}$ (DMSO- $\left.d_{6}, 500 \mathrm{MHz}\right) \delta(\mathrm{ppm})$ : 1.95, $2.24\left(3 \mathrm{H}, 2 \mathrm{~s}, 5-\mathrm{CH}_{3}\right), 6.16-7.70(7 \mathrm{H}, \mathrm{m}, \mathrm{Ar}-\mathrm{H}), 7.33,7.43$ $\left(2 \mathrm{H}, 2 \mathrm{~s}, \mathrm{SO}_{2} \mathrm{NH}_{2}\right), 10.77,10.88(1 \mathrm{H}, 2 \mathrm{~s}$, indole $\mathrm{NH})$. Anal. Calcd for $\mathrm{C}_{15} \mathrm{H}_{13} \mathrm{~N}_{3} \mathrm{O}_{3} \mathrm{~S}$ (315.34): C, 57.13, H, 4.16; N, 13.33; S, 10.17. Found: C, 56.83; H, 4.14; N, 12.82; S, 10.01.

3-[(5-Fluoro-2-oxo-1,2-dihydro-3H-indol-3-ylidene)amino]benzenesulfonamide (4c). Orange crystals, yield 43\%; m.p. 247-250 ${ }^{\circ} \mathrm{C}$; IR (KBr) $\left(\nu, \mathrm{cm}^{-1}\right): 3274,3180(\mathrm{NH}), 1731(\mathrm{C}=\mathrm{O})$, 1329, $1156(\mathrm{~S}=\mathrm{O})$; ${ }^{1} \mathrm{H}-\mathrm{NMR}$ (DMSO- $\left.d_{6}, 500 \mathrm{MHz}\right) \delta(\mathrm{ppm})$ : 5.97-7.33 (4H, m, indole $\mathrm{C}_{4,6}-\mathrm{H}$, phenyl $\left.\mathrm{C}_{2,4}-\mathrm{H}\right), 6.90,6.92(1 \mathrm{H}$, $2 \mathrm{dd}, J=8.30,4.39 \mathrm{~Hz}$, indole $\left.\mathrm{C}_{7}-\mathrm{H}\right), 7.34,7.44\left(2 \mathrm{H}, 2 \mathrm{~s}, \mathrm{SO}_{2} \mathrm{NH}_{2}\right)$, 7.48, $7.66\left(1 \mathrm{H}, 2 \mathrm{t}, J=7.80 \mathrm{~Hz}\right.$, phenyl $\left.\mathrm{C}_{5}-\mathrm{H}\right), 7.56,7.71(1 \mathrm{H}, 2 \mathrm{~d}$, $J=7.80 \mathrm{~Hz}$, phenyl $\left.\mathrm{C}_{6}-\mathrm{H}\right), 10.90,11.02(1 \mathrm{H}, 2 \mathrm{~s}$, indole $\mathrm{NH})$. Anal. Calcd for $\mathrm{C}_{14} \mathrm{H}_{10} \mathrm{FN}_{3} \mathrm{O}_{3} \mathrm{~S}$ (319.31): C, 52.66, H, 3.16; N, 13.16; S, 10.04. Found: C, 52.29; H, 3.58; N, 13.18; S, 10.38.

3-[(5-Chloro-2-oxo-1,2-dihydro-3H-indol-3-ylidene)amino]benzenesulfonamide (4d). Orange powder, yield 43\%; m.p. 252-254 ${ }^{\circ} \mathrm{C}$; IR $(\mathrm{KBr})\left(\nu, \mathrm{cm}^{-1}\right): 3284,3173(\mathrm{NH}), 1733(\mathrm{C}=\mathrm{O})$, 1325, $1148(\mathrm{~S}=\mathrm{O}) ;{ }^{1} \mathrm{H}-\mathrm{NMR}$ (DMSO- $\left.d_{6}, 500 \mathrm{MHz}\right) \delta(\mathrm{ppm})$ : 6.87-7.73 (7H, m, Ar-H), 7.35, $7.46\left(2 \mathrm{H}, 2 \mathrm{~s}, \mathrm{SO}_{2} \mathrm{NH}_{2}\right), 11.10$, $11.12(1 \mathrm{H}, 2 \mathrm{~s}$, indole $\mathrm{NH})$. Anal. Calcd for $\mathrm{C}_{14} \mathrm{H}_{10} \mathrm{ClN}_{3} \mathrm{O}_{3} \mathrm{~S}$ (335.76): C, 50.08, H, 3.00; N, 12.51; S, 9.55. Found: C, 49.96; $\mathrm{H}, 3.01 ; \mathrm{N}, 12.32 ; \mathrm{S}, 9.79$.

3-[(2-Oxo-5-(trifluoromethoxy)-1,2-dihydro-3H-indol-3-ylidene)amino]benzenesulfonamide (4e). Orange powder, yield 25\%; m.p. 205-207 ${ }^{\circ} \mathrm{C}$; IR (KBr) $\left(\nu, \mathrm{cm}^{-1}\right)$ : 3338, $3282(\mathrm{NH}), 1667$, $1733(\mathrm{C}=\mathrm{O}), 1327,1150(\mathrm{~S}=\mathrm{O}) ;{ }^{1} \mathrm{H}-\mathrm{NMR}\left(\mathrm{DMSO}-d_{6}, 500 \mathrm{MHz}\right)$ $\delta$ (ppm): $7.31\left(2 \mathrm{H}, \mathrm{s}, \mathrm{SO}_{2} \mathrm{NH}_{2}\right), 7.42-7.71(6 \mathrm{H}, \mathrm{m}, \mathrm{Ar}-\mathrm{H}), 8.13$ $\left(1 \mathrm{H}, \mathrm{s}\right.$, indole $\left.\mathrm{C}_{4}-\mathrm{H}\right), 10.19(1 \mathrm{H}, \mathrm{s}$, indole $\mathrm{NH})$. Anal. Calcd for $\mathrm{C}_{15} \mathrm{H}_{10} \mathrm{~F}_{3} \mathrm{~N}_{3} \mathrm{O}_{4} \mathrm{~S}$ (385.32): C, 46.76, H, 2.62; N, 10.91; S, 8.32. Found: C, 46.97; H, 2.32; N, 10.76; S, 8.27.

3-[(5-Nitro-2-oxo-1,2-dihydro-3H-indol-3-ylidene)amino]benzenesulfonamide (4f). Orange powder, yield 30\%; m.p. 175-178 ${ }^{\circ} \mathrm{C}$; IR (KBr) $\left(\nu, \mathrm{cm}^{-1}\right): 3311,3262(\mathrm{NH}), 1748(\mathrm{C}=\mathrm{O})$, 1307, $1154(\mathrm{~S}=\mathrm{O}) .{ }^{1} \mathrm{H}-\mathrm{NMR}$ (DMSO- $\left.d_{6}, 400 \mathrm{MHz}\right) \delta(\mathrm{ppm})$ : 7.31, $7.42\left(2 \mathrm{H}, 2 \mathrm{~s}, \mathrm{SO}_{2} \mathrm{NH}_{2}\right), 6.97-8.65(7 \mathrm{H}, \mathrm{m}, \mathrm{Ar}-\mathrm{H}), 10.19$, $11.26(1 \mathrm{H}, 2 \mathrm{~s}$, indole $\mathrm{NH})$. Anal. Calcd for $\mathrm{C}_{14} \mathrm{H}_{10} \mathrm{~N}_{4} \mathrm{O}_{5} \mathrm{~S}$ (346.32): C, 48.55, H, 2.91; N, 16.18; S, 9.26. Found: C, 48.74; H, 3.04; N, 16.18; S, 9.46.

4-[(2-Oxo-1,2-dihydro-3H-indol-3-ylidene)amino]benzenesulfonamide (5a). ${ }^{22}$ Yellow powder, yield 68\%; m.p. $278-279{ }^{\circ} \mathrm{C}$; IR $(\mathrm{KBr})\left(\nu, \mathrm{cm}^{-1}\right): 3324,3233(\mathrm{NH}), 1723,1750(\mathrm{C}=\mathrm{O})$, 1329, $1144(\mathrm{~S}=\mathrm{O})$; ${ }^{1} \mathrm{H}-\mathrm{NMR}$ (DMSO- $\left.d_{6}, 500 \mathrm{MHz}\right) \delta(\mathrm{ppm})$ : 6.33, $7.60\left(1 \mathrm{H}, 2 \mathrm{~d}, J=7.81 \mathrm{~Hz}\right.$, indole $\left.\mathrm{C}_{7}-\mathrm{H}\right), 6.73,7.06(1 \mathrm{H}, 2 \mathrm{t}$, $J=7.81 \mathrm{~Hz}$, indole $\left.\mathrm{C}_{6}-\mathrm{H}\right), 6.86,6.89(1 \mathrm{H}, 2 \mathrm{~d}, J=7.81 \mathrm{~Hz}$, indole $\left.\mathrm{C}_{4}-\mathrm{H}\right), 7.08,7.15\left(2 \mathrm{H}, 2 \mathrm{~d}, J=8.30 \mathrm{~Hz}\right.$, phenyl $\left.\mathrm{C}_{3,5}-\mathrm{H}\right)$, $7.28,7.35\left(2 \mathrm{H}, 2 \mathrm{~s}, \mathrm{SO}_{2} \mathrm{NH}_{2}\right), 7.35,7.46(1 \mathrm{H}, 2 \mathrm{t}, J=7.81 \mathrm{~Hz}$, indole $\left.\mathrm{C}_{5}-\mathrm{H}\right), 7.73,7.89\left(2 \mathrm{H}, 2 \mathrm{~d}, J=8.30 \mathrm{~Hz}\right.$, phenyl $\left.\mathrm{C}_{2,6}-\mathrm{H}\right)$, 10.89, $11.00(1 \mathrm{H}, 2 \mathrm{~s}$, indole $\mathrm{NH})$. Anal. Calcd for $\mathrm{C}_{14} \mathrm{H}_{11} \mathrm{~N}_{3} \mathrm{O}_{3} \mathrm{~S}$ (301.32): C, 55.80; H, 3.68; N, 13.95; S, 10.64. Found: C, 56.05; H, 4.05; N, 13.86; S, 10.53 . 
4-[(1-Methyl-2-oxo-1,2-dihydro-3H-indol-3-ylidene)amino]benzenesulfonamide (5b). ${ }^{23}$ Orange powder, yield $62 \%$; m.p. 252-253 ${ }^{\circ} \mathrm{C}$; IR (KBr) $\left(\nu, \mathrm{cm}^{-1}\right): 3328,3213(\mathrm{NH}), 1725(\mathrm{C}=\mathrm{O})$, 1321, $1154(\mathrm{~S}=\mathrm{O}) ;{ }^{1} \mathrm{H}-\mathrm{NMR}$ (DMSO- $\left.d_{6}, 500 \mathrm{MHz}\right) \delta(\mathrm{ppm})$ : $3.07,3.19\left(3 \mathrm{H}, 2 \mathrm{~s}, 1-\mathrm{CH}_{3}\right), 6.36,7.64(1 \mathrm{H}, 2 \mathrm{~d}, J=7.81 \mathrm{~Hz}$, indole $\left.\mathrm{C}_{7}-\mathrm{H}\right), 6.80-7.16\left(4 \mathrm{H}, \mathrm{m}\right.$, indole $\mathrm{C}_{4,6}-\mathrm{H}$, phenyl $\left.\mathrm{C}_{3,5}-\mathrm{H}\right)$, 7.30, $7.36\left(2 \mathrm{H}, 2 \mathrm{~s}, \mathrm{SO}_{2} \mathrm{NH}_{2}\right), 7.46,7.56(1 \mathrm{H}, 2 \mathrm{t}, J=7.81 \mathrm{~Hz}$, indole $\left.\mathrm{C}_{5}-\mathrm{H}\right), 7.74,7.90\left(2 \mathrm{H}, 2 \mathrm{~d}, J=8.29 \mathrm{~Hz}\right.$, phenyl $\left.\mathrm{C}_{2,6}-\mathrm{H}\right)$. Anal. Calcd for $\mathrm{C}_{15} \mathrm{H}_{13} \mathrm{~N}_{3} \mathrm{O}_{3} \mathrm{~S}$ (315.34): C, 57.13, H, 4.16; N, 13.33; S, 10.17. Found: C, 57.37; H, 4.57; N, 13.20; S, 10.34.

4-[(5-Methyl-2-oxo-1,2-dihydro-3 $\mathrm{H}$-indol-3-ylidene)amino]benzenesulfonamide (5c). ${ }^{24}$ Orange powder, yield 58\%; m.p. 265-266 ${ }^{\circ} \mathrm{C}$; IR (KBr) $\left(\nu, \mathrm{cm}^{-1}\right): 3323,3232(\mathrm{NH}), 1723,1751$ $(\mathrm{C}=\mathrm{O}), 1320,1147(\mathrm{~S}=\mathrm{O}) ;{ }^{1} \mathrm{H}-\mathrm{NMR} \quad\left(\mathrm{DMSO}-d_{6}, 500 \mathrm{MHz}\right)$ $\delta$ (ppm): 2.24, $2.27\left(3 \mathrm{H}, 2 \mathrm{~s}, 5-\mathrm{CH}_{3}\right), 5.77,6.11(1 \mathrm{H}, 2 \mathrm{~s}$, indole $\left.\mathrm{C}_{4}-\mathrm{H}\right), 6.57,6.79\left(2 \mathrm{H}, 2 \mathrm{~d}, J=8.79 \mathrm{~Hz}\right.$, phenyl $\left.\mathrm{C}_{3,5}-\mathrm{H}\right), 6.85$, $7.36\left(2 \mathrm{H}, 2 \mathrm{~s}, \mathrm{SO}_{2} \mathrm{NH}_{2}, \mathrm{D}_{2} \mathrm{O}\right.$ exch.), 7.07, $7.29(1 \mathrm{H}, 2 \mathrm{~d}, J=8.29$ $\mathrm{Hz}$, indole $\left.\mathrm{C}_{6}-\mathrm{H}\right), 7.14,7.28\left(1 \mathrm{H}, 2 \mathrm{~d}, J=8.29 \mathrm{~Hz}\right.$, indole $\left.\mathrm{C}_{7}-\mathrm{H}\right)$, 7.38, $7.43\left(2 \mathrm{H}, 2 \mathrm{~d}, J=8.79 \mathrm{~Hz}\right.$, phenyl $\left.\mathrm{C}_{2,6}-\mathrm{H}\right), 10.78,10.89$ $\left(1 \mathrm{H}, 2 \mathrm{~s}\right.$, indole $\mathrm{NH}, \mathrm{D}_{2} \mathrm{O}$ exch.). Anal. Calcd for $\mathrm{C}_{15} \mathrm{H}_{13} \mathrm{~N}_{3} \mathrm{O}_{3} \mathrm{~S}$ (315.34): C, 57.13, H, 4.16; N, 13.33; S, 10.17. Found: C, 56.89; $\mathrm{H}, 4.14 ; \mathrm{N}, 12.95 ; \mathrm{S}, 10.53$.

4-[(1,5-Dimethyl-2-oxo-1,2-dihydro-3H-indol-3-ylidene)amino]benzenesulfonamide (5d). Orange powder, yield $20 \%$; m.p. 223-225 ${ }^{\circ} \mathrm{C}$; IR (KBr) $\left(\nu, \mathrm{cm}^{-1}\right): 3325,3235(\mathrm{NH}), 1723,1753$ $(\mathrm{C}=\mathrm{O}), 1328,1147(\mathrm{~S}=\mathrm{O}) ;{ }^{1} \mathrm{H}-\mathrm{NMR} \quad\left(\mathrm{DMSO}-d_{6}, 500 \mathrm{MHz}\right)$ $\delta$ (ppm): 1.99, 2.31 (3H, 2s, 5- $\left.\mathrm{CH}_{3}\right), 3.04,3.17\left(3 \mathrm{H}, 2 \mathrm{~s}, 1-\mathrm{CH}_{3}\right)$, 6.14, $7.47\left(1 \mathrm{H}, 2 \mathrm{~s}\right.$, indole $\left.\mathrm{C}_{4}-\mathrm{H}\right), 6.98,7.07(1 \mathrm{H}, 2 \mathrm{~d}, J=8.30 \mathrm{~Hz}$, indole $\left.\mathrm{C}_{6}-\mathrm{H}\right), 7.01,7.14\left(2 \mathrm{H}, 2 \mathrm{~d}, J=8.30 \mathrm{~Hz}\right.$, phenyl $\left.\mathrm{C}_{3,5}-\mathrm{H}\right)$, 7.18, $7.28\left(1 \mathrm{H}, 2 \mathrm{~d}, J=8.30 \mathrm{~Hz}\right.$, indole $\left.\mathrm{C}_{7}-\mathrm{H}\right), 7.29,7.37(2 \mathrm{H}, 2 \mathrm{~s}$, $\left.\mathrm{SO}_{2} \mathrm{NH}_{2}\right), 7.73,7.89\left(2 \mathrm{H}, 2 \mathrm{~d}, J=8.30 \mathrm{~Hz}\right.$, phenyl $\left.\mathrm{C}_{2,6}-\mathrm{H}\right)$. Anal. Calcd for $\mathrm{C}_{16} \mathrm{H}_{15} \mathrm{~N}_{3} \mathrm{O}_{3} \mathrm{~S}$ (329.37): C, 58.34, H, 4.59; N, 12.76; S, 9.74. Found: C, 58.01; H, 4.90; N, 12.34; S, 9.65.

4-[(5-Fluoro-2-oxo-1,2-dihydro-3H-indol-3-ylidene)amino]benzenesulfonamide (5e). Orange powder, yield 20\%; m.p. 275-277 ${ }^{\circ} \mathrm{C}$; IR (KBr) $\left(\nu, \mathrm{cm}^{-1}\right):(\mathrm{C}=\mathrm{O}) ; 1 \mathrm{H}-\mathrm{NMR}$ (DMSO- $d_{6}$, $500 \mathrm{MHz}) \delta$ (ppm): 6.08, $7.52(1 \mathrm{H}, 2 \mathrm{dd}, J=7.81,4.32 \mathrm{~Hz}$, indole $\left.\mathrm{C}_{7}-\mathrm{H}\right), 6.95,6.99\left(1 \mathrm{H}, 2 \mathrm{dd}, J=8.78,2.44 \mathrm{~Hz}\right.$, indole $\mathrm{C}_{6}{ }^{-}$ $\mathrm{H}), 7.18,7.25\left(2 \mathrm{H}, 2 \mathrm{~d}, J=8.30 \mathrm{~Hz}\right.$, phenyl $\left.\mathrm{C}_{3,5}-\mathrm{H}\right), 7.34,7.40$ $\left(1 \mathrm{H}, 2 \mathrm{dd}, J=8.78,2.44 \mathrm{~Hz}\right.$, indole $\left.\mathrm{C}_{4}-\mathrm{H}\right), 7.37,7.46(2 \mathrm{H}, 2 \mathrm{~s}$, $\left.\mathrm{SO}_{2} \mathrm{NH}_{2}\right), 7.81,7.98\left(2 \mathrm{H}, 2 \mathrm{~d}, J=8.30 \mathrm{~Hz}\right.$, phenyl $\left.\mathrm{C}_{2,6}-\mathrm{H}\right), 10.97$, $11.11(1 \mathrm{H}, 2 \mathrm{~s}$, indole $\mathrm{NH})$. Anal. Calcd for $\mathrm{C}_{14} \mathrm{H}_{10} \mathrm{FN}_{3} \mathrm{O}_{3} \mathrm{~S}$ (319.31): C, 52.66, H, 3.16; N, 13.16; S, 10.04. Found: C, 52.99; H, 3.17; N, 12.90; S, 9.85 .

4-[(5-Fluoro-1-methyl-2-oxo-1,2-dihydro-3H-indol-3-ylidene)amino]benzenesulfonamide (5f). Orange powder, yield 44\%; m.p. $247-249{ }^{\circ} \mathrm{C}$; IR (KBr) $\left(\nu, \mathrm{cm}^{-1}\right): 3327,3225(\mathrm{NH}), 1734$ $(\mathrm{C}=\mathrm{O}), 1322,1155(\mathrm{~S}=\mathrm{O}) ;{ }^{1} \mathrm{H}-\mathrm{NMR} \quad\left(\mathrm{DMSO}-d_{6}, 500 \mathrm{MHz}\right)$ $\delta$ (ppm): 3.06, $3.19\left(3 \mathrm{H}, 2 \mathrm{~s}, 1-\mathrm{CH}_{3}\right), 6.03-7.52$ (5H, m, indole $\mathrm{C}_{4,6,7}-\mathrm{H}$, phenyl $\left.\mathrm{C}_{3,5}\right), 7.31,7.40\left(2 \mathrm{H}, 2 \mathrm{~s}, \mathrm{SO}_{2} \mathrm{NH}_{2}\right), 7.75,7.92$ $\left(2 \mathrm{H}, 2 \mathrm{~d}, J=8.29 \mathrm{~Hz}\right.$, phenyl $\left.\mathrm{C}_{2,6}-\mathrm{H}\right)$. Anal. Calcd for $\mathrm{C}_{15} \mathrm{H}_{12} \mathrm{FN}_{3} \mathrm{O}_{3} \mathrm{~S}$ (333.33): C, 54.05, H, 3.63; N, 12.61; S, 9.62. Found: C, 54.06; H, 3.71; N, 12.46; S, 9.70.

4-[(5-Chloro-2-oxo-1,2-dihydro-3H-indol-3-ylidene)amino]benzenesulfonamide $(5 \mathrm{~g}) \cdot{ }^{25}$ Yellow powder, yield $61 \%$; m.p. 271-272 ${ }^{\circ} \mathrm{C}$; IR (KBr) $\left(\nu, \mathrm{cm}^{-1}\right): 3330,3259(\mathrm{NH}), 1723,1745$
$(\mathrm{C}=\mathrm{O}), 1328,1151(\mathrm{~S}=\mathrm{O}) ;{ }^{1} \mathrm{H}-\mathrm{NMR} \quad\left(\mathrm{DMSO}-d_{6}, 500 \mathrm{MHz}\right)$ $\delta$ (ppm): 6.28-7.50 (3H, m, indole $\left.\mathrm{C}_{4,6,7}-\mathrm{H}\right), 7.11,7.17(2 \mathrm{H}$, $2 \mathrm{dd}, J=6.83,1.95 \mathrm{~Hz}$, phenyl $\left.\mathrm{C}_{3,5}-\mathrm{H}\right), 7.29,7.40(2 \mathrm{H}, 2 \mathrm{~s}$, $\left.\mathrm{SO}_{2} \mathrm{NH}_{2}\right), 7.74,7.91\left(2 \mathrm{H}, 2 \mathrm{dd}, J=6.83,1.95 \mathrm{~Hz}\right.$, phenyl $\left.\mathrm{C}_{2,6}-\mathrm{H}\right)$, 11.03, $11.14(1 \mathrm{H}, 2 \mathrm{~s}$, indole $\mathrm{NH})$. Anal. Calcd for $\mathrm{C}_{14} \mathrm{H}_{10} \mathrm{ClN}_{3} \mathrm{O}_{3} \mathrm{~S}$ (335.76): C, 50.08, H, 3.00; N, 12.51; S, 9.55. Found: C, 49.96; H, 3.24; N, 12.48; S, 9.20.

4-[(5-Chloro-1-methyl-2-oxo-1,2-dihydro-3H-indol-3-ylidene)amino]benzenesulfonamide $(\mathbf{5 h})$. Orange crystals, yield $40 \%$; m.p. $221-223{ }^{\circ} \mathrm{C}$; IR (KBr) $\left(\nu, \mathrm{cm}^{-1}\right): 3341,3235(\mathrm{NH}), 1678$, $1727(\mathrm{C}=\mathrm{O}), 1332,1157(\mathrm{~S}=\mathrm{O}) ;{ }^{1} \mathrm{H}-\mathrm{NMR}$ (DMSO- $\left.d_{6}, 500 \mathrm{MHz}\right)$ $\delta$ (ppm): 3.07, $3.20\left(3 \mathrm{H}, 2 \mathrm{~s}, 1-\mathrm{CH}_{3}\right), 6.30-7.62(5 \mathrm{H}, \mathrm{m}$, indole $\mathrm{C}_{4,6,7}-\mathrm{H}$, phenyl $\left.\mathrm{C}_{3,5}-\mathrm{H}\right), 7.30,7.41\left(2 \mathrm{H}, 2 \mathrm{~s}, \mathrm{SO}_{2} \mathrm{NH}_{2}\right), 7.75$, $7.92\left(2 \mathrm{H}, 2 \mathrm{dd}, J=8.78,1.95 \mathrm{~Hz}\right.$, phenyl $\left.\mathrm{C}_{2,6}-\mathrm{H}\right)$. Anal. Calcd for $\mathrm{C}_{15} \mathrm{H}_{12} \mathrm{ClN}_{3} \mathrm{O}_{3} \mathrm{~S}$ (349.79): C, 51.51, H, 3.46; N, 12.01; S, 9.17. Found: C, 49.05; H, 3.93; N, 11.56; S, 9.53.

4-[(5-Bromo-1-methyl-2-oxo-1,2-dihydro-3 $\mathrm{H}$-indol-3-ylidene)amino]benzenesulfonamide (5i). Orange crystals, yield 16\%; m.p. $235-237{ }^{\circ} \mathrm{C}$; IR (KBr) $\left(\nu, \mathrm{cm}^{-1}\right): 3336,3234(\mathrm{NH}), 1676$, $1729(\mathrm{C}=\mathrm{O}), 1331,1156(\mathrm{~S}=\mathrm{O}) ;{ }^{1} \mathrm{H}-\mathrm{NMR}\left(\mathrm{DMSO}-d_{6}, 500 \mathrm{MHz}\right)$ $\delta$ (ppm): 3.19 (3H, s, 1- $\left.\mathrm{CH}_{3}\right), 7.11(1 \mathrm{H}, 2 \mathrm{dd}, J=8.78,3.42 \mathrm{~Hz}$, indole $\left.\mathrm{C}_{6}-\mathrm{H}\right), 7.17\left(1 \mathrm{H}, \mathrm{d}, J=8.78 \mathrm{~Hz}\right.$, indole $\left.\mathrm{C}_{7}-\mathrm{H}\right), 7.30(1 \mathrm{H}$, $\mathrm{s}$, indole $\left.\mathrm{C}_{4}-\mathrm{H}\right), 7.41\left(2 \mathrm{H}, \mathrm{s}, \mathrm{SO}_{2} \mathrm{NH}_{2}\right), 7.75(2 \mathrm{H}, \mathrm{d}, J=8.30 \mathrm{~Hz}$, phenyl $\left.\mathrm{C}_{3,5}-\mathrm{H}\right), 7.92\left(2 \mathrm{H}, \mathrm{d}, J=8.30 \mathrm{~Hz}\right.$, phenyl $\left.\mathrm{C}_{2,6}-\mathrm{H}\right)$. Anal. Calcd for $\mathrm{C}_{15} \mathrm{H}_{12} \mathrm{BrN}_{3} \mathrm{O}_{3} \mathrm{~S}$ (394.24): C, 45.70, H, 3.07; N, 10.66; S, 8.13. Found: C, 45.72; H, 3.24; N, 10.96; S, 8.17.

4-[(2-Oxo-5-(trifluoromethoxy)-1,2-dihydro-3H-indol-3-ylidene)amino]benzenesulfonamide (5j). Yellow powder, yield $20 \%$; m.p. 193-195 ${ }^{\circ} \mathrm{C}$; IR (KBr) $\left(\nu, \mathrm{cm}^{-1}\right): 3370,3289,3212(\mathrm{NH})$, $1736(\mathrm{C}=\mathrm{O}), 1331,1158(\mathrm{~S}=\mathrm{O}) ;{ }^{1} \mathrm{H}-\mathrm{NMR}\left(\mathrm{DMSO}-d_{6}, 400 \mathrm{MHz}\right)$ $\delta$ (ppm): $7.19\left(2 \mathrm{H}, \mathrm{s}, \mathrm{SO}_{2} \mathrm{NH}_{2}\right), 7.65(4 \mathrm{H}, \mathrm{s}, \mathrm{Ar}-\mathrm{H}), 6.55-7.89$ $\left(3 \mathrm{H}, \mathrm{m}\right.$, indole $\left.\mathrm{C}_{4,6,7}-\mathrm{H}\right), 10.24(1 \mathrm{H}, \mathrm{s}$, indole $\mathrm{NH})$. Anal. Calcd for $\mathrm{C}_{15} \mathrm{H}_{10} \mathrm{~F}_{3} \mathrm{~N}_{3} \mathrm{O}_{4} \mathrm{~S}$ (385.32): C, 46.76, H, 2.62; N, 10.91; S, 8.32. Found: C, 46.66; H, 2.85; N, 10.78; S, 8.50.

4-[(5-Nitro-2-oxo-1,2-dihydro-3H-indol-3-ylidene)amino]benzenesulfonamide (5k). ${ }^{25}$ Orange powder, yield $15 \%$; m. p. $257-259{ }^{\circ} \mathrm{C}$; IR $(\mathrm{KBr})\left(\nu, \mathrm{cm}^{-1}\right): 3339,3263(\mathrm{NH}), 1752$ $(\mathrm{C}=\mathrm{O}), 1335,1153(\mathrm{~S}=\mathrm{O}) ;{ }^{1} \mathrm{H}-\mathrm{NMR} \quad\left(\mathrm{DMSO}-d_{6}, 400 \mathrm{MHz}\right)$ $\delta$ (ppm): 7.32, $7.43\left(2 \mathrm{H}, 2 \mathrm{~s}, \mathrm{SO}_{2} \mathrm{NH}_{2}\right), 7.05-8.37$ (7H, m, Ar-H), 11.59, $11.70(1 \mathrm{H}, 2 \mathrm{~s}$, indole $\mathrm{NH})$. Anal. Calcd for $\mathrm{C}_{14} \mathrm{H}_{10} \mathrm{~N}_{4} \mathrm{O}_{5} \mathrm{~S}$ (346.32): C, 48.55, H, 2.91; N, 16.18; S, 9.26. Found: C, 48.56; H, 3.28; N, 16.27; S, 9.56.

4-[(1-Methyl-5-nitro-2-oxo-1,2-dihydro-3H-indol-3-ylidene)amino]benzenesulfonamide (51). Yellow powder, yield 27\%; m.p. $230-232{ }^{\circ} \mathrm{C}$; IR (KBr) $\left(\nu, \mathrm{cm}^{-1}\right): 3312,3241(\mathrm{NH}), 1678$, $1741(\mathrm{C}=\mathrm{O}), 1339,1157(\mathrm{~S}=\mathrm{O}) ;{ }^{1} \mathrm{H}-\mathrm{NMR}\left(\mathrm{DMSO}-d_{6}, 500 \mathrm{MHz}\right)$ $\delta$ (ppm): 3.16, $3.20\left(3 \mathrm{H}, 2 \mathrm{~s}, 1-\mathrm{CH}_{3}\right), 7.17-8.47\left(9 \mathrm{H}, \mathrm{m}, \mathrm{SO}_{2} \mathrm{NH}_{2}\right.$ and $\mathrm{Ar}-\mathrm{H}$ ). Anal. Calcd for $\mathrm{C}_{15} \mathrm{H}_{10} \mathrm{~N}_{4} \mathrm{O}_{5} \mathrm{~S}$ (360.34): C, 50.00, H, 3.36; N, 15.55; S, 8.90. Found: C, 50.48; H, 3.84; N, 15.53; S, 9.03 .

\section{Enzyme inhibition assay}

A stopped-flow instrument (SX.18MV-R Applied Photophysics model) was used for assaying the CA-catalyzed $\mathrm{CO}_{2}$ hydration activity. ${ }^{26}$ Inhibitor and enzyme were preincubated for $15 \mathrm{~min}$ for allowing the complete formation of the enzyme-inhibitor 
adduct. $\mathrm{IC}_{50}$ values were obtained from dose response curves working at seven different concentrations of the test compound (from $0.1 \mathrm{nM}$ to $50 \mu \mathrm{M}$ ), by fitting the curves using PRISM (http://www.graphpad.com) and non-linear least squares methods, the obtained values representing the mean of at least three different determinations. The inhibition constants $\left(K_{\mathrm{I}}\right)$ were derived from the $\mathrm{IC}_{50}$ values by using the Cheng-Prusoff equation, as follows: $K_{\mathrm{I}}=\mathrm{IC}_{50} /\left(1+[\mathrm{S}] / K_{\mathrm{m}}\right)$ where [S] represents the $\mathrm{CO}_{2}$ concentration at which the measurement was carried out, and $K_{\mathrm{m}}$ the concentration of the substrate at which the enzyme activity is at half maximal. All enzymes used were recombinant, produced in E. coli as reported earlier. ${ }^{27-30}$ The concentrations of enzymes used in the assay were: hCA I, 12.4 nM; hCA II, 8.7 nM; hCA IX, 9.2 nM and hCA XII, $10.8 \mathrm{nM}$.

\section{Molecular modelling studies}

Preparation of ligand structures. The isatin structures 3, 4 and 5 were prepared in $3 \mathrm{D}$ with the MOE software package (v2013.08.02, Chemical Computing Group Inc., Montreal, Canada) and the ligands were energy minimized using a steepest-descent protocol (MMFF94x force field).

Preparation of hCA crystal structures for docking studies. The structures of hCA I (PDB: 3LXE, $1.90 \AA$ ), hCA II (PDB: 4E3D, $1.60 \AA$ ), hCA IX (PDB: 3IAI; $2.20 \AA$ ) and hCA XII (PDB: 1JD0; $1.50 \AA)$ were obtained from the protein databank. The protein atoms and the active site zinc ions were retained and all other atoms were omitted. The remaining structure was protonated using the MOE software package and subsequently the obtained structure was energy-minimized (AMBER99 force field). Finally, the obtained protein models were superposed on the hCA I structure using the backbone C $\alpha$-atoms and all $\mathrm{Zn}^{2+}$-ions, zinc-binding histidines and the overall backbone atoms superposed well (RMSD value: $1.281 \AA$ ).

Docking of the compounds into the hCA structures. The GOLD Suite software package (v5.2, CCDC, Cambridge, UK) and the ChemScore scoring function were used to dock the compounds into the hCA structures (50 dockings per ligand). The binding pocket was defined as all residues within $13 \AA$ of a centroid $(x$ : $-17.071, y$ : 35.081, 43.681; corresponding approximately to the position of the thiadiazole ring of acetazolamide in the 1JD0 structure). Position restraints were applied to the sulfur and nitrogen atoms of the acetazolamide sulfonamide tail of hCA XII (default settings) and were also applied to the other three hCA structures due to the low RMSD value of the superpositions.

\section{Conclusions}

We report here a panel of 23 new sulfonamides incorporating Schiff base moieties. They were obtained by reactions of variously substituted isatins with 2-, 3- and 4-amino-benzenesulfonamides. These new derivatives were tested as inhibitors of four physiologically relevant CA isoforms, involved in crucial physiological and pathological processes: the house-keeping cytosolic hCA I and II, as well as the transmembrane, tumorassociated hCA IX and XII, validated drug targets for theranostics for the management of hypoxic tumors. The new sulfonamides were moderate-weak hCA I/II inhibitors and highly potent, low nanomolar hCA IX/XII inhibitors. By using docking studies we also explained the differential inhibition of the four CA isoforms and the structural reasons connected with the selective inhibition of the transmembrane over the cytosolic isoforms. As a sulfonamide CA IX/XII inhibitor recently entered Phase I clinical trials for the management of metastatic solid tumors, compounds of the type reported here may be useful for designing different derivatives with such properties.

\section{Acknowledgements}

This project was in part financed by the Istanbul University Scientific Research Projects Department (Project Number: UDP-51361) and by an FP7 EU project (Dynano).

\section{Notes and references}

1 (a) C. T. Supuran, Nat. Rev. Drug Discovery, 2008, 7, 168; (b) M. Aggarwal, B. Kondeti and R. McKenna, Expert Opin. Ther. Pat., 2013, 23, 717; (c) J. Ivanova, J. Leitans, M. Tanc, A. Kazaks, R. Zalubovskis, C. T. Supuran and K. Tars, Chem. Commun., 2015, 51, 7108.

2 (a) V. Alterio, A. Di Fiore, K. D'Ambrosio, C. T. Supuran and G. De Simone, Chem. Rev., 2012, 112, 4421; (b) D. Neri and C. T. Supuran, Nat. Rev. Drug Discovery, 2011, 10, 767; (c) C. Capasso and C. T. Supuran, J. Enzyme Inhib. Med. Chem., 2015, 30, 325.

3 (a) C. T. Supuran, J. Enzyme Inhib. Med. Chem., 2013, 28, 229; (b) F. Briganti, S. Mangani, A. Scozzafava, G. Vernaglione and C. T. Supuran, J. Biol. Inorg. Chem., 1999, 4, 528; (c) G. De Simone and C. T. Supuran, J. Inorg. Biochem., 2012, 111, 117; (d) K. D’Ambrosio, S. Carradori, S. M. Monti, M. Buonanno, D. Secci, D. Vullo, C. T. Supuran and G. De Simone, Chem. Commun., 2015, 51, 302.

4 (a) M. Tanc, F. Carta, A. Scozzafava and C. T. Supuran, ACS Med. Chem. Lett., 2015, 6, 292; (b) A. Innocenti, A. Scozzafava, S. Parkkila, L. Puccetti, G. De Simone and C. T. Supuran, Bioorg. Med. Chem. Lett., 2008, 18, 2267.

5 (a) C. T. Supuran, Bioorg. Med. Chem., 2013, 21, 1377; (b) C. T. Supuran, J. Enzyme Inhib. Med. Chem., 2012, 27, 759; (c) A. Scozzafava, L. Menabuoni, F. Mincione, G. Mincione and C. T. Supuran, Bioorg. Med. Chem. Lett., 2001, 11, 575-582.

6 G. De Simone, V. Alterio and C. T. Supuran, Expert Opin. Drug Discovery, 2013, 8, 793.

7 S. Del Prete, D. Vullo, G. M. Fisher, K. T. Andrews, S. A. Poulsen, C. Capasso and C. T. Supuran, Bioorg. Med. Chem. Lett., 2014, 24, 4389. 
8 (a) P. Pan, A. B. Vermelho, G. Capaci Rodrigues, A. Scozzafava, M. E. Tolvanen, S. Parkkila, C. Capasso and C. T. Supuran, J. Med. Chem., 2013, 56, 1761; (b) L. Syrjanen, A. B. Vermelho, I. de Almeida Rodrigues, S. Corte-Real, T. Salonen, P. Pan, D. Vullo, S. Parkkila, C. Capasso and C. T. Supuran, J. Med. Chem., 2013, 56, 7372; (c) I. Nishimori, T. Minakuchi, K. Morimoto, S. Sano, S. Onishi, H. Takeuchi, D. Vullo, A. Scozzafava and C. T. Supuran, J. Med. Chem., 2006, 49, 2117.

9 F. Carta and C. T. Supuran, Expert Opin. Ther. Pat., 2013, 23, 681 .

10 (a) E. Masini, F. Carta, A. Scozzafava and C. T. Supuran, Expert Opin. Ther. Pat., 2013, 23, 705; (b) M. Bozdag, M. Ferraroni, F. Carta, D. Vullo, L. Lucarini, E. Orlandini, A. Rossello, E. Nuti, A. Scozzafava, E. Masini and C. T. Supuran, J. Med. Chem., 2014, 57, 9152; (c) M. Bozdag, M. Pinard, F. Carta, E. Masini, A. Scozzafava, R. McKenna and C. T. Supuran, J. Med. Chem., 2014, 57, 9673.

11 (a) G. De Simone, A. Scozzafava and C. T. Supuran, Chem. Biol. Drug Des., 2009, 74, 317; (b) C. Temperini, A. Innocenti, A. Scozzafava, S. Parkkila and C. T. Supuran, J. Med. Chem., 2010, 53, 850; (c) Z. H. Chohan, A. Scozzafava and C. T. Supuran, J. Enzyme Inhib. Med. Chem., 2003, 18, 259.

12 (a) F. Carta, A. Scozzafava and C. T. Supuran, Expert Opin. Ther. Pat., 2012, 22, 747; (b) C. T. Supuran, Expert Opin. Emerging Drugs, 2012, 17, 11.

13 (a) N. Krall, F. Pretto, W. Decurtins, G. J. L. Bernardes, C. T. Supuran and D. Neri, Angew. Chem., Int. Ed.., 2014, 53, 4231; (b) P. C. McDonald, J. Y. Winum, C. T. Supuran and S. Dedhar, Oncotarget, 2012, 3, 84; (c) F. E. Lock, P. C. McDonald, Y. Lou, I. Serrano, S. C. Chafe, C. Ostlund, S. Aparicio, J. Y. Winum, C. T. Supuran and S. Dedhar, Oncogene, 2013, 32, 5210; (d) L. Dubois, K. Douma, C. T. Supuran, R. K. Chiu, M. A. M. J. van Zandvoort, S. Pastoreková, A. Scozzafava, B. G. Wouters and P. Lambin, Radiother. Oncol., 2007, 83, 367.

14 (a) P. Swietach, S. Wigfield, P. Cobden, C. T. Supuran, A. L. Harris and R. D. Vaughan-Jones, J. Biol. Chem., 2008, 283, 20473; (b) V. Alterio, M. Hilvo, A. Di Fiore, C. T. Supuran, P. Pan, S. Parkkila, A. Scaloni, J. Pastorek, S. Pastorekova, C. Pedone, A. Scozzafava, S. M. Monti and G. De Simone, Proc. Natl. Acad. Sci. U. S. A., 2009, 106, 16233.

15 J. Pan, J. Lau, F. Mesak, N. Hundal, M. Pourghiasian, Z. Liu, F. Benard, S. Dedhar, C. T. Supuran and K. S. Lin, J. Enzyme Inhib. Med. Chem., 2014, 29, 249.

16 ClinicalTrails.gov: Safety Study of SLC-0111 in Subjects With Advanced Solid Tumours - Full Text View - ClinicalTrials_gov.mht.
17 (a) R. McKenna and C. T. Supuran, Carbonic anhydrase inhibitors drug design, in Carbonic Anhydrase: Mechanism, regulation, Links to Disease, and Industrial Applications, ed. R. McKenna and S. Frost, Springer Verlag, Heidelberg, 2014, pp. 291-323 (Subcell. Biochem. 2014, 75, 291); (b) C. T. Supuran, M. A. Ilies and A. Scozzafava, Eur. J. Med. Chem., 1998, 33, 739; (c) A. Thiry, J. M. Dognè, B. Masereel and C. T. Supuran, Curr. Top. Med. Chem., 2007, 7, 855.

18 (a) C. Capasso and C. T. Supuran, J. Enzyme Inhib. Med. Chem., 2014, 29, 379; (b) B. P. Mahon, A. M. Hendon, J. M. Driscoll, G. M. Rankin, S. A. Poulsen, C. T. Supuran and R. McKenna, Bioorg. Med. Chem., 2015, 23, 849.

19 (a) C. T. Supuran, A. Nicolae and A. Popescu, Eur. J. Med. Chem., 1996, 31, 431; (b) L. Puccetti, G. Fasolis, D. Vullo, Z. H. Chohan, A. Scozzafava and C. T. Supuran, Bioorg. Med. Chem. Lett., 2005, 15, 3096.

20 (a) G. Nasr, E. Petit, C. T. Supuran, J. Y. Winum and M. Barboiu, Bioorg. Med. Chem. Lett., 2009, 19, 6014; (b) G. Nasr, E. Petit, D. Vullo, J. Y. Winum, C. T. Supuran and M. Barboiu, J. Med. Chem., 2009, 52, 4853; (c) M. Y. Abdelrahim, M. Tanc, J. Y. Winum, C. T. Supuran and M. Barboiu, Chem. Commun., 2014, 50, 8043.

21 P. Selvam, M. Chandramohan, E. De Clercq, M. Witvrouw and C. Pannecouque, Eur. J. Pharm. Sci., 2001, 14, 313.

22 M. Hassan, Z. H. Chohan, A. Scozzafava and C. T. Supuran, J. Enzyme Inhib. Med. Chem., 2004, 19, 263.

23 Z. H. Chohan, A. U. Shaikh and M. M. Naseer, Appl. Organomet. Chem., 2006, 20, 729.

24 V. A. Muthukumar, H. C. Nagaraj, D. Bhattacherjee and S. George, Int. J. Pharm. Pharm. Sci., 2013, 5, 95.

25 S. Ramachandran and V. Uma Maheswari, Int. J. Pharm. Biosci., 2011, 2, 251.

26 R. G. Khalifah, J. Biol. Chem., 1971, 246, 2561.

27 (a) A. Sahin, S. Isik, O. Arslan, C. T. Supuran and O. Ozensoy Guler, J. Enzyme Inhib. Med. Chem., 2015, 30, 224; (b) Y. Akbaba, A. Akincioglu, H. Göçer, S. Göksu, I. Gülçin and C. T. Supuran, J. Enzyme Inhib. Med. Chem., 2014, 29, 35.

28 F. Liu, A. Martin-Mingot, F. Lecornué, A. Maresca, S. Thibaudeau and C. T. Supuran, J. Enzyme Inhib. Med. Chem., 2012, 27, 886.

29 (a) S. Del Prete, V. De Luca, D. Vullo, A. Scozzafava, V. Carginale, C. T. Supuran and C. Capasso, J. Enzyme Inhib. Med. Chem., 2014, 29, 532; (b) V. De Luca, S. Del Prete, C. T. Supuran and C. Capasso, J. Enzyme Inhib. Med. Chem., 2015, 30, 277; (c) A. Mastrolorenzo, S. Rusconi, A. Scozzafava, G. Barbaro and C. T. Supuran, Curr. Med. Chem., 2007, 14, 2734.

30 V. Alterio, M. Tanc, J. Ivanova, R. Zalubovskis, I. Vozny, S. M. Monti, A. Di Fiore, G. De Simone and C. T. Supuran, Org. Biomol. Chem., 2015, 13, 4064. 